\title{
Traumatic aortic aneurysm diagnosed by computed tomography angiography in a 22-year-old man presenting with progressive dyspnea and dysphagia
}

\author{
Xiang $\mathrm{Ma}^{1} \cdot$ Yi-Tong $\mathrm{Ma}^{1} \cdot$ Ai-Hong Liu ${ }^{1} \cdot$ Yan-Lin Wang ${ }^{2}$
}

Received: 25 April 2016 / Accepted: 2 May 2016 / Published online: 21 May 2016

(C) The Author(s) 2016. This article is published with open access at Springerlink.com

\begin{abstract}
Traumatic aortic aneurysm is a rare and potentially fatal complication that can occur secondary to acute chest trauma. However, early diagnosis of this complication is often neglected due to the presence of severe chest and abdominal trauma. We report a case of delayed traumatic aortic aneurysm that was diagnosed by computed tomography angiography on day 13 after a traffic accident.
\end{abstract}

Keywords Traumatic aortic aneurysm · Computed tomography angiography $\cdot$ Dyspnea $\cdot$ Dysphagia

\section{Abbreviations \\ CTA Computed tomography angiography \\ CT Computed tomography}

\section{Case report}

A 22-year-old man was injured in a traffic accident. His chief complaint was chest, abdominal, and back pain as well as chest tightness but experienced no loss of consciousness. He was admitted to the emergency department. A full-body computed tomography (CT) scan revealed severe blunt organ injuries and multiple fractures in his limbs, multiple rib, and lumbar vertebral bodies. On

Xiang Ma

maxiangxj@yeah.net

1 Department of Cardiology, First Affiliated Hospital of Xinjiang Medical University, 137 Liyushan Road, Ürümqi 830054, China

2 Department of Imaging Center, First Affiliated Hospital of Xinjiang Medical University, 137 Liyushan Road, Ürümqi 830054, China posttraumatic day 3 , the patient began exhibiting progressive dyspnea and dysphagia that were not improved by subsequent treatments. On posttraumatic day 13, he was transferred to our hospital for further diagnosis and treatment. Computed tomography angiography (CTA) of the chest and abdomen revealed a ruptured traumatic aortic aneurysm at the aortic isthmus with formation of a large mediastinal hematomas that severely compressed the trachea and esophagus (Fig. 1a, b). The patient died on the day after admission to our hospital.

\section{Discussion}

It remains unclear when and for how long delayed aneurysm formation occurs after trauma. But the high likelihood of traumatic aortic aneurysm should be considered in any survivor of chest contusion. And it requires prompt diagnosis and treatment.

Due to unique anatomical features, blunt aortic injuries typically occur at the aortic isthmus distal to the left subclavian artery $[1,2]$ (Fig. 1c). The esophagus and trachea are both located in the middle mediastinum, close to the aortic isthmus [3]. Therefore, compression or shifting of the trachea and esophagus are the two most reliable indications of traumatic aortic aneurysm [3]. Once the aneurysm has enlarged or has ruptured to form hematomas that compress the trachea and esophagus, progressive dyspnea and dysphagia will be observed. In our case, typical symptoms of progressive dyspnea and dysphagia caused by compression of the trachea and esophagus were present on the third day after the traffic accident. However, physicians ignored these symptoms and did not consider traumatic aortic aneurysm as a possible diagnosis. This lack of consideration led to missing the optimal timing for surgery and ultimately to the patient's death. 

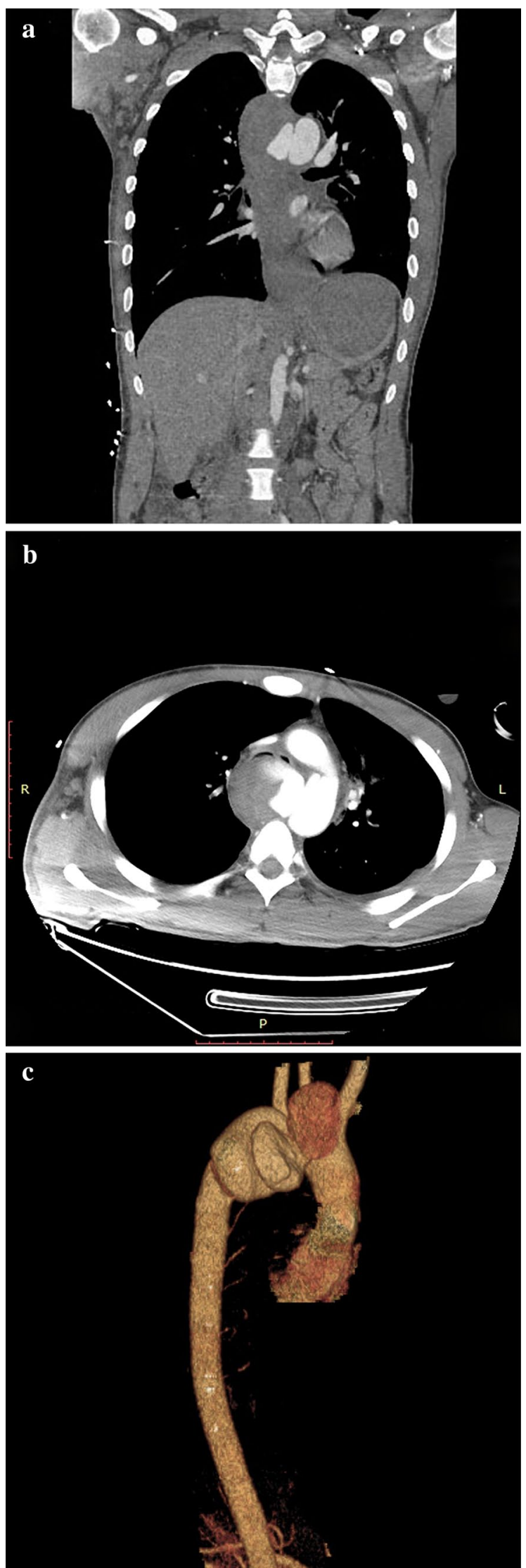

४ Fig. 1 A CT coronary reconstruction (a) revealed a traumatic aortic aneurysm with a large mediastinal hematoma that severely compressed the trachea and esophagus. A CT axial image (b) revealed a ruptured aortic aneurysm. The initial site of the left and right main bronchus, the esophagus and adjacent right lung tissue are compressed. A volumerendered image (c) shows traumatic aortic aneurysm in classic location of the aortic isthmus

In the evaluation of patients with acute chest trauma, CTA has $100 \%$ sensitivity for assessing degrees of aortic aneurysms [1] and can reveal blood vessels, their adjacent structures, and related lesions [4]. Therefore, CTA plays important roles in early diagnosis and follow-up in case of traumatic aortic aneurysm. If available, CTA should be more widely used in the assessment of traumatic aortic aneurysm.

\section{Conclusion}

We conclude that in patients surviving acute chest trauma, even when no findings an aortic aneurysm, as time goes on, physicians need a high index of suspicion for potential aortic injury in patients [2]. In particular, this injury should be considered for patients who present with typical late clinical manifestations of progressive dyspnea and dysphagia. The early diagnosis of delayed aortic aneurysm is based on repeated physical examination aided by CTA should be performed. Early diagnosis and treatment is the key to optimize patient outcome.

\section{Complaince with ethical standards}

\section{Conflict of interest None.}

Open Access This article is distributed under the terms of the Creative Commons Attribution 4.0 International License (http://creativecommons.org/licenses/by/4.0/), which permits unrestricted use, distribution, and reproduction in any medium, provided you give appropriate credit to the original author(s) and the source, provide a link to the Creative Commons license, and indicate if changes were made.

\section{References}

1. Macura KJ, Corl FM, Fishman EK, Bluemke DA (2003) Pathogenesis in acute aortic syndromes: aortic aneurysm leak and rupture and traumatic aortic transection. AJR Am J Roentgenol 181:303-307

2. Matteucci ML, Rescigno G, Altamura G, Manfrin M, D’Alfonso A et al (2006) Delayed traumatic aortic cusp detachment mimicking aortic dissection. Ann Thorac Surg 82:1093-1095

3. Gerlock AJ Jr, Muhletaler CA, Coulam CM, Hayes PT (1980) Traumatic aortic aneurysm: validity of esophageal tube displacement sign. AJR Am J Roentgenol 135:713-718

4. Cullen EL, Lantz EJ, Johnson CM, Young PM (2014) Traumatic aortic injury: CT findings, mimics, and therapeutic options. Cardiovasc Diagn Ther 4:238-244 